\title{
Effect of background ionization on plasma ignition dynamics
}

Cite as: Phys. Plasmas 24, 033503 (2017); https://doi.org/10.1063/1.4977805

Submitted: 17 December 2016 . Accepted: 15 February 2017 . Published Online: 03 March 2017

Y. Qiu, Y. Xian, (D) X. Lu, and K. Ostrikov
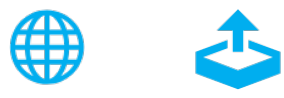

View Online

Export Citation

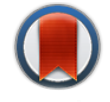

\section{ARTICLES YOU MAY BE INTERESTED IN}

Guided ionization waves: The physics of repeatability

Applied Physics Reviews 5, 031102 (2018); https://doi.org/10.1063/1.5031445

Visible light effects in plasma plume ignition

Physics of Plasmas 24, 043502 (2017); https://doi.org/10.1063/1.4979263

Perspective: The physics, diagnostics, and applications of atmospheric pressure low temperature plasma sources used in plasma medicine

Journal of Applied Physics 122, 020901 (2017); https://doi.org/10.1063/1.4993710 


\title{
Effect of background ionization on plasma ignition dynamics
}

\author{
Y. Qiu, ${ }^{1}$ Y. Xian, ${ }^{1, a)}$ X. Lu, ${ }^{1,2, a)}$ and K. Ostrikov ${ }^{3,4}$ \\ ${ }^{1}$ State Key Laboratory of Advanced Electromagnetic Engineering and Technology, Huazhong University of \\ Science and Technology, Wuhan, Hubei 430074, People's Republic of China \\ ${ }^{2}$ IFSA Collaborative Innovation Center, Shanghai Jiao Tong University, Shanghai 200240, \\ People's Republic of China \\ ${ }^{3}$ School of Chemistry, Physics and Mechanical Engineering, Queensland University of Technology, Brisbane, \\ QLD 4000, Australia \\ ${ }^{4}$ CSIRO-QUT Joint Sustainable Processes and Devices Laboratory, Commonwealth Scientific and Industrial \\ Research Organization, P.O. Box 218, Lindfield, NSW 2070, Australia
}

(Received 17 December 2016; accepted 15 February 2017; published online 3 March 2017)

The influence of background ionization on the ignition dynamics of the pulsed plasma plume is studied. The ignition delay time of each pulse is investigated by recording the voltage signal and the light emission signal. By changing the frequency, the relationship between the pulse-off time and the ignition delay time is revealed. This indicates that residual active species produced in the previous discharge play a role in the next one. With the decrease in the frequency, both time delay and ignition delay time increase. This is due to the decay of the reactive species densities in the pulse-off time. Lower concentrations of these species lead to a longer ignition delay time. The functions for calculating the ignition delay time are utilized to explain the effect of residual species. The independent data of each discharge also evidence the impact of the previous pulse. The exotic relationship between the ignition delay times of the first two pulses may be due to the electrode configuration used in this work. For a pin-to-plane electrode structure, the active species produced during the breakdown are accumulated around the anode (pin-point) where the discharge initiated for the asymmetrical electric field distribution. Published by AIP Publishing. [http://dx.doi.org/10.1063/1.4977805]

\section{INTRODUCTION}

\section{A. Streamer propagation}

Non-equilibrium atmospheric pressure plasma jets (N-APPJs), also commonly named "guided streamers," have been studied for more than 10 years ${ }^{1-8}$ owing to numerous applications such as plasma medicine, ${ }^{9-15}$ chemistry,,${ }^{5,16}$ and nanotechnology. ${ }^{17,18}$ One significant difference between the guided streamers and traditional streamers ${ }^{19,20}$ is their propagation behavior. The guided streamers propagate in a repeatable mode in the form of a "plasma bullet" traditional streamers normally propagate randomly. In other words, traditional streamers do not travel the same distance after the same delay time, as described by the original steamer discharge theories going back to the early 1900s. ${ }^{26}$

Streamer discharges emerge when a strong electric field is applied to a gas. Few initial electrons could be due to natural processes such as cosmic rays, radioactive decay, or pre-ionization, or due to residual electrons from the previous discharge for the case of high-frequency discharges. These electrons are accelerated by the strong electric field. When the electric field approaches the breakdown threshold, the electrons gain enough energy between collisions to ionize the gas, removing an electron from the atom. At the breakdown electric field, there is a balance between the production and losses of electrons. Only when the electric field exceeds the breakdown threshold, the number of electrons starts to grow exponentially, and an electron avalanche forms. The

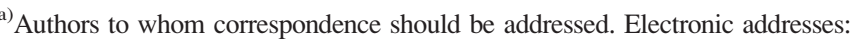
yubin.xian@hotmail.com and luxinpei@hotmail.com
}

electron avalanches leave positive ions behind, so more and more space charge builds up with time. Eventually, the electric field from all the space charge becomes comparable to the background electrical field. This is often referred to as the "avalanche to streamer transition." 27

It takes several nanoseconds for this avalanche process to develop from the initial few electrons to an electron density of the order of $10^{8} / \mathrm{cm}^{3},{ }^{27}$ when the local electric field induced by positive ions is comparable to the external applied electric field. It is noticeable that negative oxygen ions $\mathrm{O}_{2}^{-}$play an important role in providing free electrons by detachment reactions, and the field for electron detachment from $\mathrm{O}_{2}^{-}$is similar to the breakdown field. ${ }^{28}$

Electron detachment from negative ions from background ionization is the main source of seed electrons. In air, detachment reactions can occur when $\mathrm{O}_{2}^{-}$collides with neutral gas particles. The level of background ionization lies normally within $10^{3}-10^{4} / \mathrm{cm}^{3}$ considering the natural radioactivity, which is the value determined by the equilibrium between the ionization and recombination process. $^{28}$ In pulsed discharges, ions can accumulate in a sealed vessel in repetitive discharges. According to theoretical estimates, a background ionization level of about $10^{7} / \mathrm{cm}^{3}$ can exist for a gap of a few centimeters in air at atmospheric pressure at $1 \mathrm{~Hz}$ repetition rate. ${ }^{29}$

\section{B. Streamer repeatability}

In contrast to traditional streamers, which propagate in a stochastic manner, guided streamers exhibit high repeatability. ${ }^{1}$ The N-APPJs propagate in a form of plasma bullets 
along a predetermined path - the gas jet axis. To understand the high repeatability of the N-APPJs, a lot of works have recently been carried out. ${ }^{28,30,31} \mathrm{Wu}$ et al. studied the effect of the repetition rate of applied voltage on the repeatability of plasma bullets and found that when the frequency is lower than a certain threshold, the plasma bullets become unrepeatable. ${ }^{30}$ Nijdam et al. studied the streamer trail at different frequencies and found that the streamer channels do not follow the paths of the previous discharge for repetition rates up to $10 \mathrm{~Hz}^{28}$ They also studied double-pulse streamers by varying the pulse-to-pulse interval. They found that with the increase in the interval, the second-pulse streamers become less dependent on the first-pulse streamers. ${ }^{31}$

On the other hand, the effects of the background ionization level on the traditional streamers have been studied by many works. ${ }^{29,32-34}$ Chen et al. studied the influence of the frequency on the inception cloud and steamer emergence and found that more streamers separate from the inception cloud under inhomogeneous background ionization conditions at higher frequencies. ${ }^{32}$ Pancheshnyi et al. simulated the effect of photo-ionization and background ionization on propagation of streamers. $^{29,33,34}$ They found that in artificial air (mixture gas of $80 \% \mathrm{~N}_{2}$ and $20 \% \mathrm{O}_{2}$ molecules) photoionization dominates for the streamer propagation when the background ionization level is below $10^{10} / \mathrm{cm}^{3}$. This background density can be associated with a repetition frequency of $1 \mathrm{kHz}$ according to the estimates in previous works. However, in nitrogen with $1 \mathrm{ppm}$, oxygen photo-ionization still dominates up to background ionization levels of $10^{7} / \mathrm{cm}^{3}$. Meanwhile, detachment induced by background ionization is mostly a localized effect due to the high critical electric field for detachment. This effect leads to a steep decrease in the electron density around the streamer head in simulation with background ionization only.

According to all the works discussed above, it is reasonable to conclude that the repeatability of streamers might be related to the level of background ionization, which is caused by residual ions from the previous discharge or background radioactivity. The detachment reactions play a critical role in enhancing the background ionization by generating free electrons.

Besides, there are two effects that may induce random streamer modes. One is the randomness of the ignition process and the other one is the randomness of the propagation process. Most of the previous works have focused on the latter process ${ }^{24,35-37}$ whereas the streamer ignition process ${ }^{38-41}$ has attracted less attention. The ignition delay time is a critical characteristic for better understanding of the influence of the background ionization level on the repetitive behavior of the streamers. Considering the good insulating property of air at atmospheric pressure, a relatively high breakdown voltage is required for the discharge. However, when pulsed direct current (DC) voltage is used to drive the discharge, when the voltage is high, due to the long rise time of the pulse and the voltage fluctuation when the voltage achieves the peak value, it is difficult to accurately decide the starting point of the delay time. Thus low gas pressures, consequently low voltages, are needed for accurately measuring the ignition delay time.

\section{Aims and organization of the paper}

In this work, we investigate the role of background ionization in the ignition process powered by the pulsed voltage power supply with the repetition rate ranging from 1 to $1000 \mathrm{~Hz}$ in artificial air. In Section II, the experimental setup and the process parameters are described. Section III presents the results of this work and these results are discussed in Section IV. A brief summary of this work is provided in Section V.

\section{EXPERIMENTAL}

Figure 1 shows the schematic of the experiment setup used in this work. The needle-plane discharge is ignited in a chamber connected with a gas cylinder and a pumping system. A stainless steel needle is connected to the pulsed voltage power supply, and a $5 \mathrm{k} \Omega$ current limiting resistor is added to the ground terminal to prevent excessive currents. The distance $(d)$ between the needle and the plane is $20 \mathrm{~mm}$. A photomultiplier tube (PMT) is placed outside the chamber to capture the light emitted by the discharge around the pin-point of the needle through the optical window. The gas filled in the chamber is artificial air, which consists of $80 \%$ nitrogen and $20 \%$ oxygen. The gas pressure is maintained at $500 \mathrm{~Pa}$ in this experiment. The gas in the chamber is replaced after each discharge to ensure that the residual charged particles and active species have been eliminated. The pulsed voltage applied to the needle is $1 \mathrm{kV}$ with a pulse width of $5 \mu \mathrm{s}$. The frequency of the pulsed voltage varies from 1 to $1000 \mathrm{~Hz}$.

Figure 2 shows the schematic of the pulse sequence and the corresponding PMT signals. The time point $t=0$ represents the time when the first voltage pulse is applied. Here, $t_{d}$ represents the time delay when the first discharge is ignited. After the first discharge is ignited, the PMT signals can be captured in each of the following voltage pulses. The intervals $\Delta t_{1}, \Delta t_{2}$, and $\Delta t_{3}$ represent the ignition delay times of the PMT signal after the corresponding voltage pulse (80\% maximum of voltage). The interval $\Delta t_{\infty}$ represents the ignition delay time after many discharge pulses (more than 50 pulses).

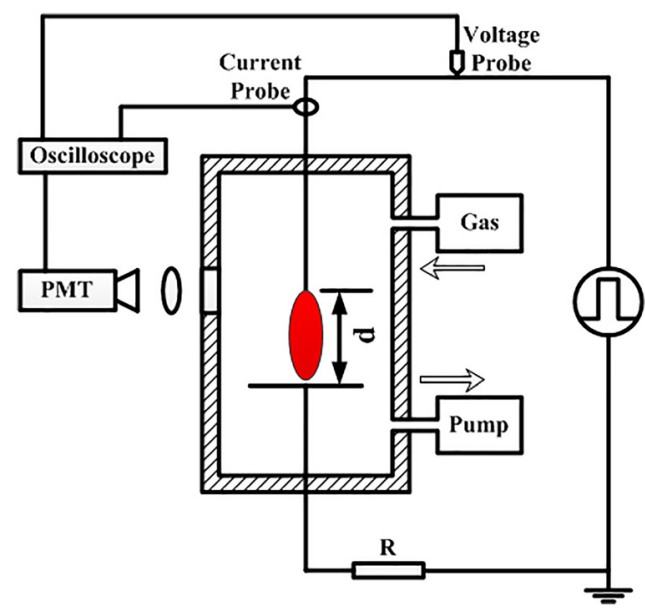

FIG. 1. Schematic of the experimental setup. 


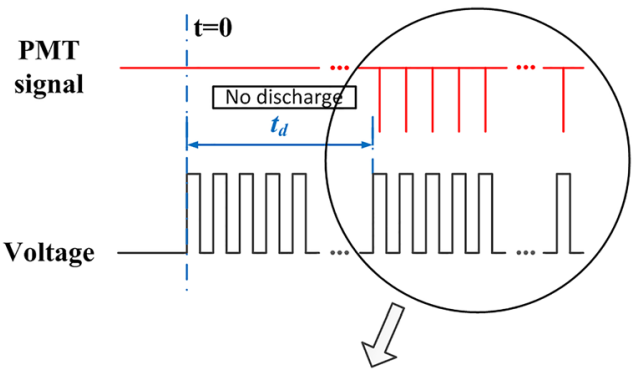

PMT

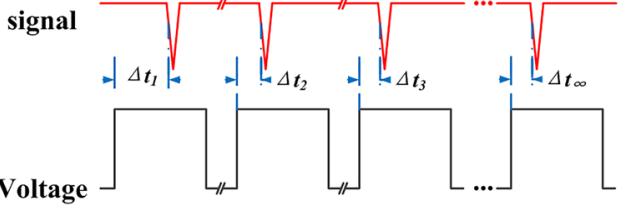

FIG. 2. Schematic of the pulse sequence and the corresponding PMT signal.

\section{EXPERIMENTAL RESULTS}

\section{A. Time delay}

As soon as the first voltage pulse is applied, the oscilloscope starts recording both the voltage signal and PMT signal until the first PMT waveform is captured, which corresponds to the ignition of the first discharge. The time interval between the first voltage pulse and the ignition of the first discharge is referred to as the time delay $\left(t_{d}\right)$ of discharge. It varies from several milliseconds to hundreds of seconds depending on the frequency of the voltage pulse.

The effect of frequency of the applied voltage pulse on the time delay $t_{d}$ of the first discharge pulse is shown in Fig. 3. The error bar in the figure represents the standard deviation of the data repeated for ten times. When the frequency is lower than $5 \mathrm{~Hz}$, no discharge is ignited even after the voltage pulses are applied for $15 \mathrm{~min}$. In general, the time delay increases with the frequency decrease. The mean value of $t_{d}$ at $1000 \mathrm{~Hz}$ is $100 \mathrm{~ms}$, which means that the first discharge is actually ignited after about 100 voltage pulses have been applied. As the frequency decreases to $5 \mathrm{~Hz}$, the

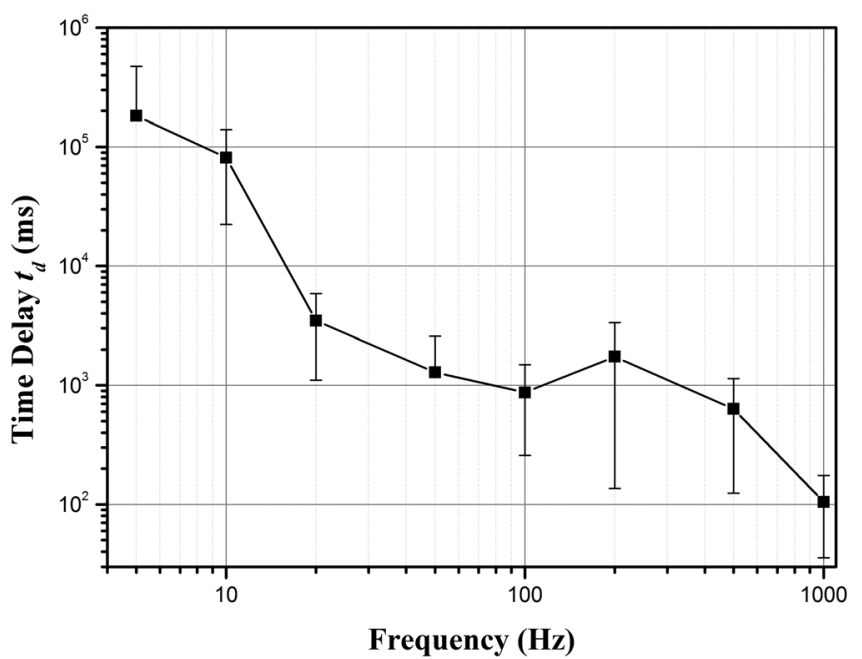

FIG. 3. The effect of frequency of the applied voltage pulse on the time delay $\mathrm{t}_{\mathrm{d}}$. Operating gas: $500 \mathrm{~Pa}$ air. mean value of time delay is $182 \mathrm{~s}$, which is three orders of magnitude longer than the delay at $1000 \mathrm{~Hz}$.

On the other hand, if we consider the number of voltage pulses before the first discharge is ignited, it is about one hundred to one thousand pulses for all the frequencies. The difference of the number of pulses before the first discharge is ignited is insignificant for all the frequencies tested. In other words, under the experimental conditions reported in this paper, the number of pulses before the first discharge is ignited depends weakly on the frequency of the applied voltage.

\section{B. Ignition delay time}

To further investigate the ignition process, the ignition delay time $\Delta t_{n}(n=1-5)$, which is defined as the time between the rising edge of the voltage pulse corresponding to the first discharge and the PMT signal waveform is detected (as illustrated in Fig. 2), are measured. As shown in Fig. 4, the $x$-axis represents the sequence of the number of discharges (from the first to the fifth discharge pulses). It is worth emphasizing that the discharges are always ignited for each voltage pulse after the first discharge. The $x$ positions of each point in Fig. 4 are intentionally shifted slightly for better clarity.

The mean value of the ignition delay time of the first discharge $\Delta t_{1}$ is several microseconds for all the frequencies used in this work. This value is close to the pulse width,
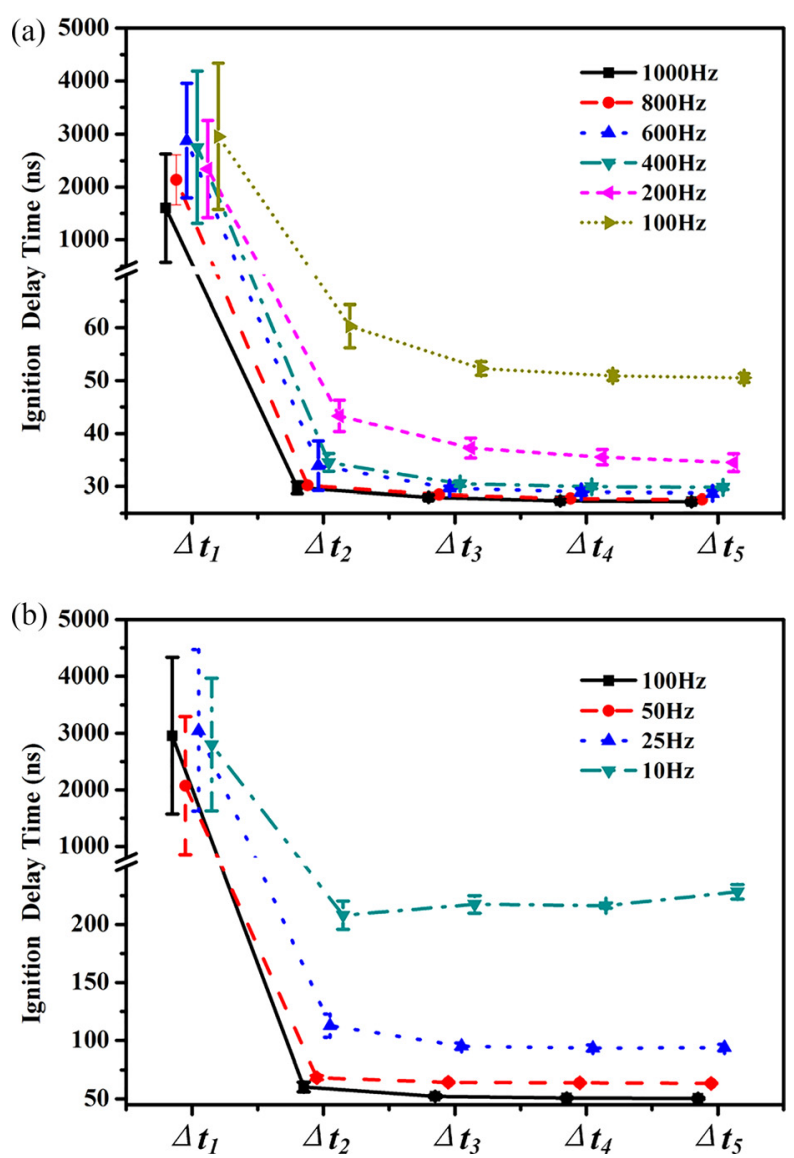

FIG. 4. The effect of frequency on the ignition delay time for the first to the fifth discharge pulses. The frequency of the applied voltage pulse varies between (a) 1000 and $100 \mathrm{~Hz}$ and (b) 100 and $10 \mathrm{~Hz}$. 
which is five microseconds. On the other hand, the mean values of $\Delta t_{2}$ to $\Delta t_{5}$ are much shorter than $\Delta t_{1}$. Despite the decreasing trend between $\Delta t_{2}$ and $\Delta t_{5}$, there is no significant difference among $\Delta t_{2}$ to $\Delta t_{5}$ for the same frequency of the voltage pulse. The variation of $\Delta t_{n}$ decreases with the increase of $n$, where $n$ is the sequence number of the discharge pulse. Furthermore, for different frequencies, the delay times $\Delta t_{2}$ to $\Delta t_{5}$ show an obvious difference. At higher frequencies, the value of $\Delta t_{n}(n=2-5)$ is reduced. The mean values of $\Delta t_{2}$ are about 28,50 , and $210 \mathrm{~ns}$ for the frequencies $1000 \mathrm{~Hz}, 100 \mathrm{~Hz}$, and $10 \mathrm{~Hz}$, respectively.

Additionally, it is worth emphasizing that the variation of $\Delta t_{1}$, which is of the order of a microsecond, is much higher than $\Delta t_{n}(n=2-5)$ for all the frequencies tested. It means that the breakdown occurs randomly during the first discharge and stabilizes for the following pulses. With the decrease in the frequency, the variation of $\Delta t_{n}(n=2-5)$ slightly increases.

To further understand the effect of the frequency of the applied voltage on the delay time of the stabilized discharge states (more than 100 pulses), the delay time $\Delta t_{\infty}$ of the discharges in the stable states is measured for different frequencies. As shown in Fig. 5, with the increase in the frequency, both $\Delta t_{\infty}$ and its variation decrease significantly. When the frequency is higher than $100 \mathrm{~Hz}, \Delta t_{\infty}$ reaches a constant value of about $50 \mathrm{~ns}$. Taking into account the delay of the PMT detector, the actual ignition delay time is less than $50 \mathrm{~ns}$. The variation of the ignition delay time $\Delta t_{\infty}$ is less than $1 \mathrm{~ns}$ when the frequency is higher than $20 \mathrm{~Hz}$. The value of $\Delta t_{\infty}$ increases to $6,20,49$, and $145 \mathrm{~ns}$ when the frequency decreases to $10,5,2$, and $1 \mathrm{~Hz}$, respectively. In other words, the discharge becomes unrepeatable with the frequency decrease.

\section{The relation between $\Delta t_{1}$ and $\Delta t_{2}$}

It is worth pointing out that there is some correlation between $\Delta t_{1}$ and $\Delta t_{2}$ for each discharge. Figure 6 shows the obtained $\Delta t_{1}$ and $\Delta t_{2}$ for twenty discharges. For the discharge where $\Delta t_{1}$ is high, $\Delta t_{2}$ is also relatively high for the corresponding discharge.

When $\Delta t_{l}$ is less than $4000 \mathrm{~ns}, \Delta t_{2}$ increases almost linearly with the increase of $\Delta t_{l}$. On the other hand, when $\Delta t_{l}$ is higher than $4000 \mathrm{~ns}, \Delta t_{2}$ increases much faster and is

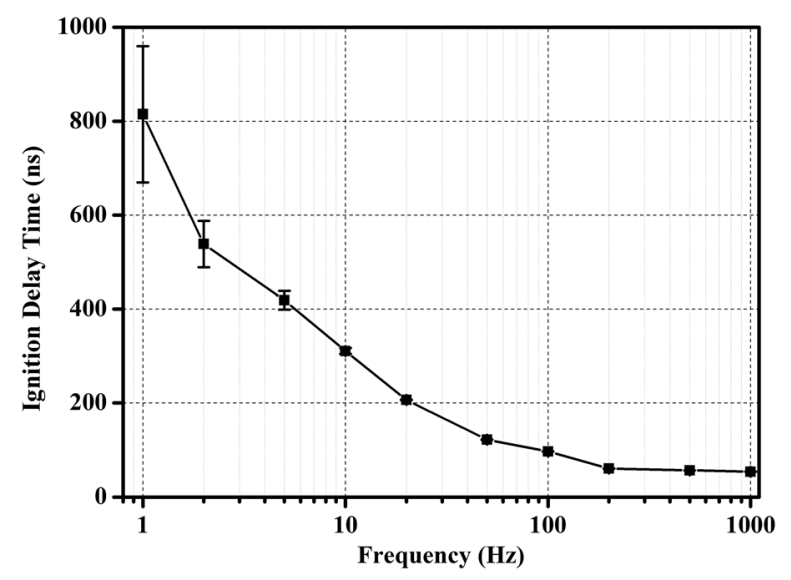

FIG. 5. The effect of frequency of the applied voltage on $\Delta t_{\infty}$.

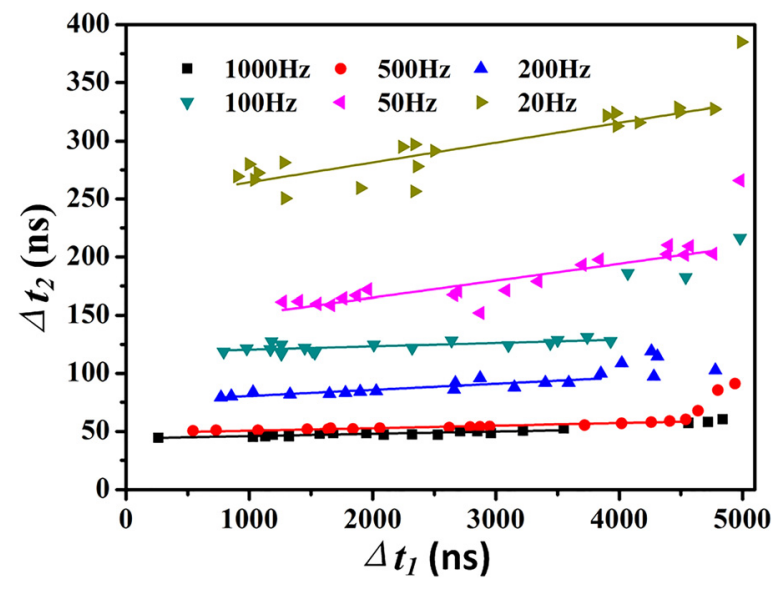

FIG. 6. The relation between $\Delta \mathrm{t}_{1}$ and $\Delta \mathrm{t}_{2}$ for each discharge at different frequencies.

scattered over a broad range. For example, at $500 \mathrm{~Hz}, \Delta t_{2}$ rises from 50 to $60 \mathrm{~ns}$ when $\Delta t_{l}$ increases from 500 to $4000 \mathrm{~ns}$ and then it increases to $90 \mathrm{~ns}$ when $\Delta t_{1}$ increases further to about $4900 \mathrm{~ns}$. Additionally, it shows that with the increase in the frequency, the values of $\Delta t_{2}$ become less scattered. This indicates that there is a strong "memory effect" at higher frequencies. ${ }^{42}$

\section{DISCUSSION}

It can be seen that the frequency of the applied voltage has a great influence on the discharge ignition process. The background ionization level, remaining from the previous discharge, is enhanced with the increase in frequency $f$. Before the discharge occurs, the level of background ionization solely due to the ionization caused by the cosmic rays is estimated to be only of the order of $10^{3}-10^{4} / \mathrm{cm}^{3}$. When the first discharge is ignited, much higher concentrations of charged particles and also excited species, especially metastable state species, are present. Most of them then decay with time through various pathways, including recombination, de-excitation, and some others. With the increase in the frequency, the time between the nearby pulses decreases. Consequently, the decay time becomes short, which results in higher concentrations of these charged particles and reactive species. These species can directly or indirectly provide the seed electrons for the ignition of the next discharge, which results in shorter ignition times.

\section{A. Ignition delay time}

As shown in Figure 7, considering direct current (DC) condition, ignition delay time $t_{d c}$ consists of two components: statistical time $t_{s}$ and formation time $t_{f} .{ }^{41}$ (The statement of $t_{d c}$ can also be adopted in defining ignition delay time $\Delta t_{n}(\mathrm{n}=1,2,3 \ldots)$ for each pulse can be seen as a DC voltage applied for a short time.) The first component, $t_{s}$, presents the time interval from the voltage-on to the moment when sufficiently energetic seed electrons can produce an electron avalanche. The second component, $t_{f}$, represents the time for the seed electrons to induce the discharge to convert the working gas from an insulating to a conducting state. 


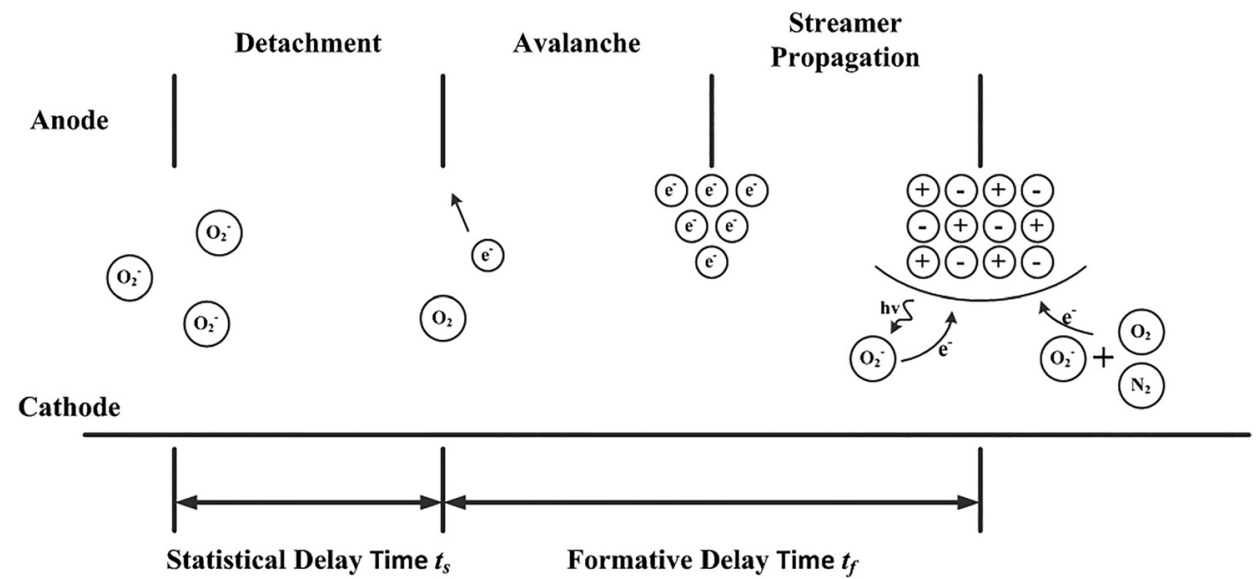

FIG. 7. Schematic diagram of the discharge process.
By the theoretical derivation process, the average value and deviation of the statistical time $t_{s}$ can be obtained from the following equations: ${ }^{40}$

$$
\begin{aligned}
\overline{t_{s}} & =1 /(Y \cdot W), \\
\sigma & =1 /(Y \cdot W),
\end{aligned}
$$

where $Y$ is the appearance rate of an initial free electron and $\mathrm{W}$ is the probability of breakdown caused by a free electron. The value of $Y$ is determined by the gas type and the concentration of charged and neutral active species. The breakdown probability $W$ can be seen as constant at the same voltage and electrode structure. Although the precise value of $t_{s}$ cannot be calculated by the equations, how the statistical time $t_{s}$ changes can be predicted qualitatively by analyzing the change in the value of $Y$ and $W$.

The second component of the ignition delay time is the formation time $t_{f}$, which can be seen as constant for the same conditions. The distribution of the ignition delay time $t_{d c}$ depends on the ratio of $t_{s}$ and $t_{f}$. Three cases are considered here:

$t_{f} \gg t_{s}$. It corresponds to the case with very large $Y$, which means that the $t_{d c}$ is dominated by $t_{f}$.

(ii) $t_{f} \approx t_{s}$. The formation time and statistical time are of the same order.

(iii) $t_{f} \ll t_{s}$. In this case, the $Y$ is small enough and the ignition delay time $t_{d c}$ is mainly due to the statistical time $t_{s}$.

Considering the appearance rate of an initial free electron $Y$, the electrons can be produced through background ionization and photo-ionization. However, before the discharge occurs, there is no light emission from the gap, which means that the photo-ionization effect can be neglected here. This is why the background ionization is considered to be the main source of the initial free electrons. The electron detachment process takes place in the region where the electric field surpasses the critical value

$$
\begin{aligned}
& \mathrm{O}_{2}^{-}+\mathrm{O}_{2} \rightarrow \mathrm{e}+\mathrm{O}_{2}+\mathrm{O}_{2}, \\
& \mathrm{O}_{2}^{-}+\mathrm{N}_{2} \rightarrow \mathrm{e}+\mathrm{N}_{2}+\mathrm{O}_{2} .
\end{aligned}
$$

The detachment time $\tau_{D}$ is estimated to be several nanoseconds to hundreds of nanoseconds depending on the electric field strength. ${ }^{38}$ The value of $\tau_{D}$ decreases with the increase in the strength of the electric field as shown in Fig. 8.

Both Equations (3) and (4) represent the second-order reaction. Considering the very small ionization rates in lowtemperature plasmas, the concentration of $\mathrm{O}_{2}$ and $\mathrm{N}_{2}$ molecules can be assumed constant. Hence, reactions (3) and (4) can be considered as the first-order reactions. In other words, higher $\mathrm{O}_{2}^{-}$concentrations lead to the faster reactions. Consequently, the appearance rate $Y$ increases with a stronger electric field and a higher density of negative oxygen ions. Subsequently, the statistical delay time $t_{s}$ will become shorter.

\section{B. Effect of frequency on time delay and ignition delay time}

Before the discharge occurs, the gas in the chamber can be considered as the insulator with an extremely low concentration of ions and neutral active species in it. The ion density in the chamber is estimated to be $10^{3}-10^{4} / \mathrm{cm}^{3}$ as mentioned in Section I. The seed electrons are initiated by cosmic rays, which leads to the very low values of $Y$. Since $W=$ const at a given voltage, according to Ref. $40 \overline{t_{s}} \gg t_{f}$. The ignition delay time at DC condition $t_{d c}$ can be expressed as $t_{d c} \approx \overline{t_{s}}=1 /(Y \cdot W)$ as well as the deviation of it. In this case, the fluctuation of cosmic rays is insignificant and the values of $t_{d c}$ and $\sigma$ remain stable within the measurement times. We recall that the ignition delay time at DC conditon $t_{d c}$ here means the period of time needed for a discharge to

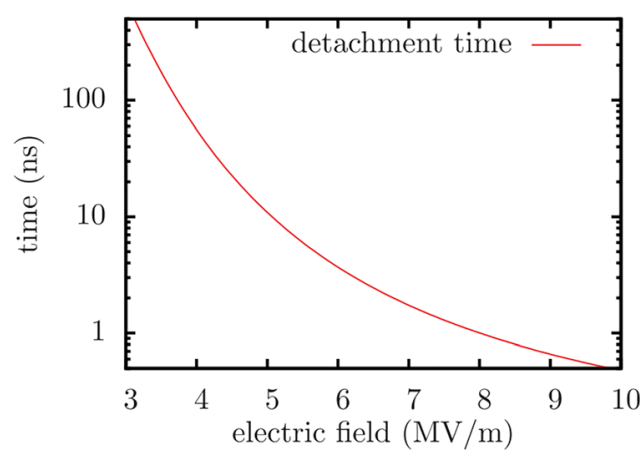

FIG. 8. The detachment time $\tau_{\mathrm{D}}$ as a function of the electric field strength in air. In stronger fields, negative ions have a higher energy and drift faster, so they are more likely to lose an electron in a collision with a neutral molecule. ${ }^{38}$ 
occur after a DC voltage is turned on. In the pulsed voltage case, the total voltage-on time before the breakdown can be seen as $t_{d c}$. Thus, the $t_{d c}$ can be seen as break up into several time intervals equal to the pulse width of the pulse voltage ( $5 \mu \mathrm{s}$ in this work). Therefore, the average value and the deviation of the number of pulse periods should be stable and very close with the change in the frequency, which agrees well with the results shown in Figure 3. The numbers of the pulse periods before the breakdown occurs are of the order of several hundred when the frequency is higher than $10 \mathrm{~Hz}$. So with the decrease in the frequency, the period becomes longer, and as a consequence, the time delay $t_{d}$ in this work increases.

As mentioned above, the ignition delay time of the first several discharge periods in Figure 4 can also be resolved. Before the breakdown occurs, the average value and deviation of $\Delta t_{l}$ are very close for a small $Y$ value. While as the discharge occurs between the electrodes, a large number of negative oxygen ions are produced in the chamber, which leads to a higher appearance rate $Y$ in the next pulse. So the ignition delay time of the following discharges $\Delta t_{2}-\Delta t_{5}$ is much shorter than $\Delta t_{l}$. On the other hand, with the accumulation of negative oxygen ions between the pulses, the value of $Y$ increases faster in subsequent discharges, which leads to a lower value of $t_{s}$. In this case $\Delta t_{2}-\Delta t_{5}$ exhibits a decreasing trend. Furthermore, the reason why a lower frequency condition leads to a higher $\Delta t_{2}-\Delta t_{5}$ value is similar to the case of $\Delta t_{\infty}$.

The effect of the discharge frequency on the ignition delay time after many periods $\left(\Delta t_{\infty}\right)$ is shown in Figure 5. The period of time $\Delta t_{\infty}$ changes little at high-frequency $(>100 \mathrm{~Hz})$ conditions and then increases gradually with a decrease in the frequency. The plateau region of $\Delta t_{\infty}$ with a short ignition delay time is due to the presence of numerous ions in the gas left from the previous discharge. This effect can significantly reduce the statistical time through the generation of more free electrons.

As shown in Table I, the rate coefficients of the recombination reaction between ions and electrons are around $2 \times 10^{-7}$ $\mathrm{cm}^{3} / \mathrm{s}$. The concentrations of positive ions are estimated to be $10^{9} / \mathrm{cm}^{3}$ according to previous work. ${ }^{33}$ Thus, the recombination time $\tau_{r}$ is estimated to be $\tau_{r}=\left(\mathrm{k} \cdot C_{i o n}\right)^{-1}=5 \times 10^{-3} s$ $=5 \mathrm{~ms}$. This means that for the case of the pulse interval time less than $5 \mathrm{~ms}$, the concentration of residual ions is much higher than in the case of longer pulse-off time. These ions can provide free electrons efficiently during the next pulse. Thus, the

TABLE I. Reaction rate coefficients of the electron-ion recombination and ion-ion recombination. ${ }^{43}$

\begin{tabular}{lcc}
\hline \hline & Reaction & Rate coefficient $\left(\mathrm{cm}^{3} / \mathrm{s}\right)$ \\
\hline Electron-ion recombination & $\mathrm{e}+\mathrm{N}_{2}^{+} \rightarrow \mathrm{N}+\mathrm{N}$ & $3 \times 10^{-8}$ \\
& $\mathrm{e}+\mathrm{O}_{2}^{+} \rightarrow \mathrm{O}+\mathrm{O}$ & $3 \times 10^{-8}$ \\
& $\mathrm{e}+\mathrm{O}_{4}^{+} \rightarrow \mathrm{O}_{2}+\mathrm{O}_{2}$ & $3 \times 10^{-7}$ \\
Ion-ion recombination & $\mathrm{O}^{-}+\mathrm{O}^{+} \rightarrow \mathrm{O}+\mathrm{O}$ & $2.7 \times 10^{-7}$ \\
& $\mathrm{O}^{-}+\mathrm{N}^{+} \rightarrow \mathrm{O}+\mathrm{N}$ & $2.6 \times 10^{-7}$ \\
& $\mathrm{O}^{-}+\mathrm{O}_{2}^{+} \rightarrow \mathrm{O}+\mathrm{O}_{2}$ & $1 \times 10^{-7}$ \\
& $\mathrm{O}_{2}^{-}+\mathrm{O}^{+} \rightarrow \mathrm{O}_{2}+\mathrm{O}_{2}$ & $2 \times 10^{-7}$ \\
& $\mathrm{O}_{2}^{-}+\mathrm{O}_{2}^{+} \rightarrow \mathrm{O}_{2}+\mathrm{O}_{2}$ & $4.2 \times 10^{-7}$ \\
& $\mathrm{O}_{2}^{-}+\mathrm{N}_{2}^{+} \rightarrow \mathrm{O}_{2}+\mathrm{N}_{2}$ & $1.6 \times 10^{-7}$ \\
\hline \hline
\end{tabular}

appearance rate of electrons $Y$ is relatively high that the statistical time $t_{s}$ is much less than the formation time $t_{f}$ as shown in case (i). As we can see, the ignition delay time is approximately equal to the formation time $\left(t_{d} \approx t_{f}\right)$, which changes little in this work.

As the time interval between the two discharge pulses increases, the concentration of residual ions decreases, which leads to a lower background ionization level. Since $t_{f} \ll t_{s}$ (case iii), the ignition delay time $t_{d}$ can be expressed as $t_{d} \approx t_{s}=1 /(Y \cdot W)$. Since $W$ is stable with a given voltage, $Y$ depends on the concentration of residual active species and decreases at lower background ionization levels. So the $\Delta t_{\infty}$, as well as the variation of $\Delta t_{\infty}$, increases for lower pulse frequencies as shown in Figure 5.

\section{Further discharge investigation}

Recently, most researches on discharge ignition delay time have been performed using statistical methods. Few studies focus on what happens once the discharge is on. In this work, the effect of the previous discharge on the following one is measured precisely. In Figure 6 , the influence of $\Delta t_{1}$ on $\Delta t_{2}$ is presented. It can be seen that a larger $\Delta t_{1}$ value can induce a larger $\Delta t_{2}$ value. It is due to the generation of positive species and active species during the discharge period. After the breakdown occurs, positive ions and metastable atoms are created by intense collisions among electrons, atoms, and ions. These reactions continue until the beginning of the falling edge of the pulsed voltage. With a given pulse width $(5 \mu \mathrm{s}$ in this work), larger $\Delta t_{1}$ induces a shorter reaction time $t_{c}=T_{o n}-\Delta t_{1}$ as shown in Figure 9(a) (Figures 9(a) and 9(b) are just concept image for the convenience of explanations rather than any kind of models), which will further induce a lower concentration of $\mathrm{O}_{2}^{-}\left(n_{\text {ion, },}\right)$. These species decay during the same time period $T_{\text {off }}$ and influence the ignition delay time of the next pulse. As shown in Figure 9(b) (arrow 1), when $n_{\text {ion, },}$ is higher, the value of $n_{i o n, l}$ is higher after the same period of time $T_{\text {off. }}$ Lower concentrations of these species induce a smaller $Y$ value. Likewise, the ignition delay time $\Delta t_{2}$ gets larger with the constant $W$ value. Thus, higher $\Delta t_{1}$ corresponds to higher $\Delta t_{2}$. Considering the influence of background ionization level on the production of free electrons and the lifetime of these species in this work, we can confirm that $\Delta t_{1}$ has an impact on $\Delta t_{2}$ at frequencies $100-1000 \mathrm{~Hz}$. And that is the reason why the value of $\Delta t_{2}$ at these frequencies changes only about $10 \mathrm{~ns}$ when $\Delta t_{1}<4000 \mathrm{~ns}$.

With an even higher $\Delta t_{1}(>4000 \mathrm{~ns})$, the value of $\Delta t_{2}$ rises more rapidly compared to the case of $\Delta t_{1}<4000 \mathrm{~ns}$ as shown in Figure 6. It may be due to the fact that ions are mainly produced at the beginning of the discharge. These species are accumulated rapidly and then increase at a relatively slower rate in the pulse-on time as shown in Figure 9(a). Therefore, if the first discharge occurs closely to the falling edge, the number of species will be much lower for the short accumulation time $t_{c}$ between the breakdowns and the voltage-off moments. The ignition delay time of the secondary discharge will be much larger due to the lower concentration of these species, which leads to a smaller $Y$ value. More research is needed to validate this hypothesis. 


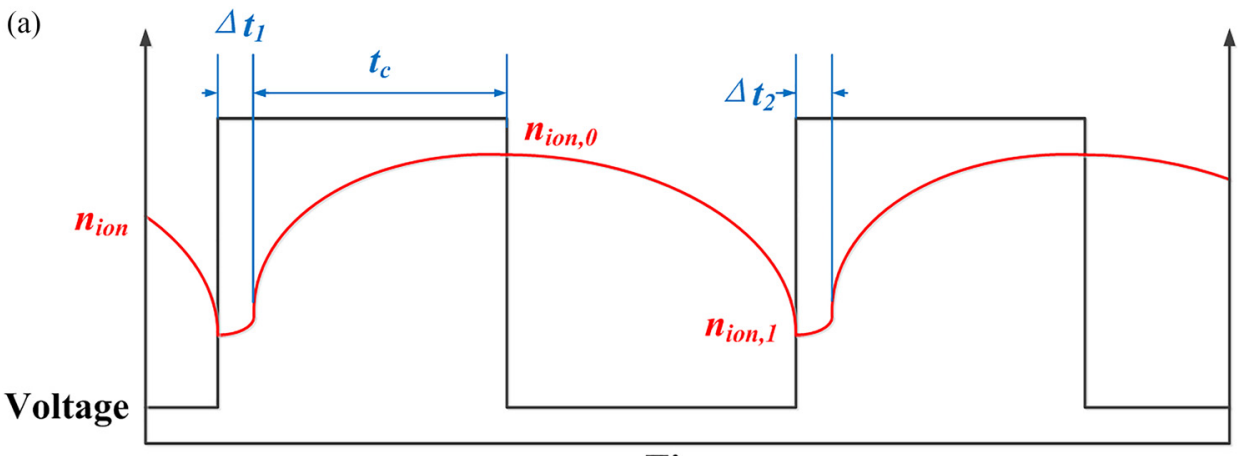

Time

(b)

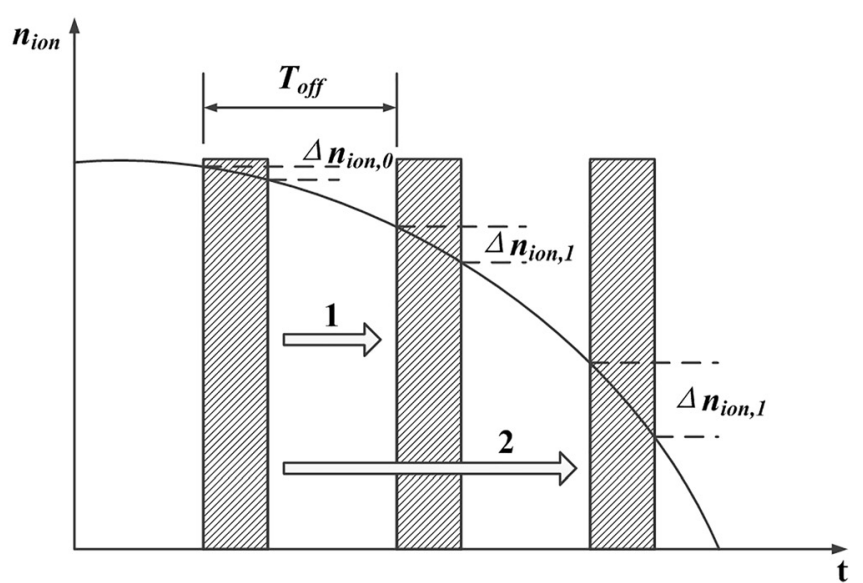

FIG. 9. The schematic of the change in the concentration of ions with time. Here, $\mathrm{n}_{\text {ion }}$ represents the total concentration of these species. (a) The change of $n_{\text {ion }}$ versus time. (b) The decay of $\mathrm{n}_{\text {ion }}$ in pulse-off time.
Another exotic phenomenon revealed from Figure 6 is that the influence of $\Delta t_{1}$ on $\Delta t_{2}$ is more significant with the decrease in the frequency. Depending on the memory effect, the residual species produced by the previous discharge will have a weaker impact on the next discharge as these species decay during the time between the two discharges. However, in this work, the slopes of the fitting curves of each frequency present an increasing trend with the decrease in the frequency as shown in Figure 6. This trend may be due to the pin-to-plane electrode structure used in this work. After the previous discharge occurs, a high concentration of $\mathrm{O}_{2}^{-}$is produced around the anode, i.e., the sharp pin-point where the electric field is much stronger. ${ }^{44}$ Consequently, the reactions are much more intense in that area. In the following pulseoff time, the decay of these species takes place mostly outside of this area and then extends to the center. So the concentration of these species around the anode decreases slowly at the initial time of $T_{\text {off }}$ and then accelerates with time. As shown in Figure 9(b) (arrow 1 for the high repetition rate and arrow 2 for the low repetition rate), for the same $\Delta n_{\text {ion, } 0}, \Delta n_{\text {ion, } 1}$ becomes larger after a relatively longer period of time $T_{\text {off. }}$ Likewise, larger $\Delta n_{\text {ion,l }}$ can further lead to the much larger differences of the ignition delay time in subsequent discharges. Thus, the influence of $\Delta t_{1}$ on $\Delta t_{2}$ is more obvious at lower frequencies.

\section{CONCLUSION}

In summary, the effects of frequency on time delay and ignition delay time of pulsed plasma discharges ignited in a pin-to-plane configuration have been investigated. Both the time delay before the breakdown and the ignition delay time of each pulse voltage increase with the frequency decrease. The residual species produced in previous discharges have a significant influence on the subsequent discharges. Based on Equations (1)-(4), the effect of the residual species on the ignition delay time has been discussed. For short $T_{\text {off }}$ periods of time, the residual negative oxygen ions enhance the production of free electrons and significantly reduce the statistical delay time $t_{s}$ of the next pulse. The ignition delay time is approximately equal to the formation time $t_{f}$, which changes little at the same conditions. For long $T_{\text {off }}$ periods of time, the background ionization level has mostly decreased through the recombination effect. The ignition delay time thus corresponds to cases (ii) and (iii). The statistical delay time increases at lower ion densities.

Further investigation has focused on each single discharge rather than statistical methods used before. The relationship between the ignition delay times of the first two pulses has been revealed. The linear relationship between $\Delta t_{1}$ and $\Delta t_{2}$ in a single discharge shows the effect of residual species produced in the previous discharge. Larger $\Delta t_{1}$ induces larger $\Delta t_{2}$, which indicates that less residual species are produced and the concentration of these species is relatively low when the next pulse comes. For the case of $\Delta t_{l}>4000 \mathrm{~ns}$, the value of $\Delta t_{2}$ increases much faster when $\Delta t_{1}$ increases. This may be because the ions are mainly produced at the very early discharge stages as shown in Figure 9(a).

Additionally, lower frequency leads to a greater influence of $\Delta t_{1}$ on $\Delta t_{2}$ as shown in Figure 6. This extraordinary result may be attributed to the structure of the electrodes. The pinto-plane structure causes the concentration of residual species 
to decrease slowly at the beginning of the pulse-off time as shown in Figure 9(b). This effect leads to stronger interactions between the two successive discharges and more research is warranted to prove this interesting possibility.

\section{ACKNOWLEDGMENTS}

This work was partially supported by the National Natural Science Foundation (Grant Nos. 51507071, 51277087, 51477066, 51625701, and 11505070), CSIRO Science Leadership Scheme, and the Australian Research Council.

${ }^{1}$ X. Lu, G. V. Naidis, M. Laroussi, and K. Ostrikov, Phys. Rep. 540, 123 (2014).

${ }^{2}$ A. Schutze, J. Y. Jeong, S. E. Babayan, J. Park, G. S. Selwyn, and R. F. Hicks, IEEE Trans. Plasma Sci. 26, 1685 (1998).

${ }^{3}$ S. Wu, X. Lu, D. Liu, Y. Yang, Y. Pan, and K. Ostrikov, Phys. Plasmas 21, 103508 (2014)

${ }^{4}$ X. Lu, G. V. Naidis, M. Laroussi, S. Reuter, D. B. Graves, and K. Ostrikov, Phys. Rep. 630, 1 (2016).

${ }^{5}$ T. Murakami, K. Niemi, T. Gans, D. O'Connell, and W. G. Graham, Plasma Sources Sci. Technol. 22, 15003 (2013).

${ }^{6}$ F. Iza, J. K. Lee, and M. G. Kong, Phys. Rev. Lett. 99, 75004 (2007).

${ }^{7}$ V. Sarron, E. Robert, S. Dozias, M. Vandamme, D. Ries, and J. M. Pouvesle, IEEE Trans. Plasma Sci. 39, 2356 (2011).

${ }^{8}$ B. Bruneau, T. Gans, D. O'Connell, A. Greb, E. V. Johnson, and J.-P. Booth, Phys. Rev. Lett. 114, 125002 (2015).

${ }^{9}$ W. Tian and M. J. Kushner, J. Phys. D: Appl. Phys. 47, 165201 (2014).

${ }^{10}$ G. Y. Park, S. J. Park, M. Y. Choi, I. G. Koo, J. H. Byun, J. W. Hong, J. Y. Sim, G. J. Collins, and J. K. Lee, Plasma Sources Sci. Technol. 21, 43001 (2012).

${ }^{11}$ S. Reuter, H. Tresp, K. Wende, M. U. Hammer, J. Winter, K. Masur, A. Schmidt-Bleker, and K.-D. Weltmann, IEEE Trans. Plasma Sci. 40, 2986 (2012).

${ }^{12}$ A. Shashurin, M. Keidar, S. Bronnikov, R. A. Jurjus, and M. A. Stepp, Appl. Phys. Lett. 93, 181501 (2008).

${ }^{13}$ M. Laroussi, IEEE Trans. Plasma Sci. 37, 714 (2009).

${ }^{14}$ T. von Woedtke, S. Reuter, K. Masur, and K. D. Weltmann, Phys. Rep. 530, 291 (2013).

${ }^{15}$ M. Laroussi and X. Lu, Appl. Phys. Lett. 87, 113902 (2005).

${ }^{16}$ D. B. Graves, J. Phys. D: Appl. Phys. 45, 263001 (2012).

${ }^{17}$ S. A. Norberg, E. Johnsen, and M. J. Kushner, J. Appl. Phys. 118, 13301 (2015).
${ }^{18}$ K. Ostrikov, E. C. Neyts, and M. Meyyappan, Adv. Phys. 62, 113 (2013).

${ }^{19}$ H. S. Uhm, Y. S. Byeon, K. B. Song, E. H. Choi, H.-Y. Ryu, and J. Lee, Phys. Plasmas 17, 113510 (2010).

${ }^{20}$ G. V. Naidis, J. Phys. D: Appl. Phys. 38, 3889 (2005).

${ }^{21}$ X. Lu and M. Laroussi, J. Appl. Phys. 100, 63302 (2006).

${ }^{22}$ N. Mericam-Bourdet, M. Laroussi, A. Begum, and E. Karakas, J. Phys. D: Appl. Phys. 42, 55207 (2009).

${ }^{23}$ A. Shashurin, M. N. Shneider, and M. Keidar, Plasma Sources Sci. Technol. 21, 34006 (2012).

${ }^{24}$ G. V. Naidis, J. Phys. D: Appl. Phys. 43, 402001 (2010).

${ }^{25}$ X. Lu, M. Laroussi, and V. Puech, Plasma Sources Sci. Technol. 21, 34005 (2012).

${ }^{26}$ H. S. Uhm, E. H. Choi, and G. S. Cho, Appl. Phys. Lett. 78, 592 (2001).

${ }^{27}$ M. Rabie and C. M. Franck, J. Phys. D: Appl. Phys. 49, 175202 (2016).

${ }^{28}$ S. Nijdam, G. Wormeester, E. M. van Veldhuizen, and U. Ebert, J. Phys. D: Appl. Phys. 44, 455201 (2011).

${ }^{29}$ S. Pancheshnyi, Plasma Sources Sci. Technol. 14, 645 (2005).

${ }^{30}$ S. Wu, X. Lu, and Y. Pan, Curr. Appl. Phys. 13, S1 (2013).

${ }^{31}$ S. Nijdam, E. Takahashi, A. H. Markosyan, and U. Ebert, Plasma Sources Sci. Technol. 23, 25008 (2014).

${ }^{32}$ S. Chen, L. C. J. Heijmans, R. Zeng, S. Nijdam, and U. Ebert, J. Phys. D: Appl. Phys. 48, 175201 (2015).

${ }^{33}$ G. Wormeester, S. Pancheshnyi, A. Luque, S. Nijdam, and U. Ebert, J. Phys. D: Appl. Phys. 43, 505201 (2010).

${ }^{34}$ S. V. Pancheshnyi, S. M. Starikovskaia, and A. Y. Starikovskii, J. Phys. D: Appl. Phys. 34, 105 (2001)

${ }^{35}$ C.-W. Lo and S. Hamaguchi, J. Phys. D: Appl. Phys. 44, 375201 (2011).

${ }^{36}$ T. Ito, T. Kanazawa, and S. Hamaguchi, Phys. Rev. Lett. 107, 65002 (2011).

${ }^{37}$ T. Ito, K. Kobayashi, U. Czarnetzki, and S. Hamaguchi, J. Phys. D: Appl. Phys. 43, 62001 (2010).

${ }^{38}$ A. Sun, J. Teunissen, and U. Ebert, J. Phys. D: Appl. Phys. 47, 445205 (2014).

${ }^{39}$ A. A. Ponomarev and N. L. Aleksandrov, Plasma Sources Sci. Technol. 24, 35001 (2015)

${ }^{40}$ M. M. Pejovic, G. S. Ristic, and J. P. Karamarkovic, J. Phys. D: Appl. Phys. 35, R91 (2002).

${ }^{41}$ W. Pfeiffer, IEEE Trans. Electr. Insul. EI-17, 505 (1982).

${ }^{42}$ M. M. Pejović, B. J. Mijović, and D. A. Bošan, J. Phys. D: Appl. Phys. 16, L149 (1983).

${ }^{43}$ A. Fridman and L. A. Kennedy, Plasma Physics and Engineering, 2nd ed. (CRC, 2011).

${ }^{44}$ R. Ono, C. Tobaru, Y. Teramoto, and T. Oda, Plasma Sources Sci. Technol. 18, 25006 (2009). 